\title{
Autophagy Induced by Ischemic Preconditioning is Essential for Cardioprotection
}

\author{
Chengqun Huang $\cdot$ Smadar Yitzhaki $\cdot$ \\ Cynthia N. Perry • Wayne Liu • Zoltan Giricz • \\ Robert M. Mentzer Jr. • Roberta A. Gottlieb
}

Received: 19 January 2010 / Accepted: 22 April 2010 /Published online: 11 May 2010

(C) The Author(s) 2010. This article is published with open access at Springerlink.com

\begin{abstract}
Based on growing evidence linking autophagy to preconditioning, we tested the hypothesis that autophagy is necessary for cardioprotection conferred by ischemic preconditioning (IPC). We induced IPC with three cycles of 5 min regional ischemia alternating with 5 min reperfusion and assessed the induction of autophagy in mCherry-LC3 transgenic mice by imaging of fluorescent autophagosomes in cryosections. We found a rapid and significant increase in the number of autophagosomes in the risk zone of the preconditioned hearts. In Langendorff-perfused hearts subjected to an IPC protocol of $3 \times 5 \mathrm{~min}$ ischemia, we also observed an increase in autophagy within $10 \mathrm{~min}$, as assessed by Western blotting for p62 and cadaverine dye binding. To establish the role of autophagy in IPC cardioprotection, we inhibited autophagy with Tat-ATG5 $5^{\mathrm{K} 130 \mathrm{R}}$, a dominant negative mutation of the autophagy protein Atg5. Cardioprotection by IPC was reduced in rat hearts perfused
\end{abstract}

\footnotetext{
C. Huang $\cdot$ S. Yitzhaki $\cdot$ C. N. Perry $\cdot$ W. Liu $\cdot$ Z. Giricz $\cdot$

R. M. Mentzer Jr. R. A. Gottlieb $(\bowtie)$

BioScience Center, San Diego State University,

5500 Campanile Drive,

San Diego, CA 92182-4650, USA

e-mail: robbieg@sciences.sdsu.edu

C. N. Perry

Molecular Pathology Graduate Program,

University of California San Diego,

San Diego, CA, USA

R. M. Mentzer Jr.

Cardiovascular Research Institute,

Wayne State University School of Medicine,

Detroit, MI, USA
}

with recombinant Tat-ATG5 ${ }^{\mathrm{K} 130 \mathrm{R}}$. To extend the potential significance of autophagy in cardioprotection, we also assessed three structurally unrelated cardioprotective agents - UTP, diazoxide, and ranolazine - for their ability to induce autophagy in HL-1 cells. We found that all three agents induced autophagy; inhibition of autophagy abolished their protective effect. Taken together, these findings establish autophagy as an end-effector in ischemic and pharmacologic preconditioning.

Keywords Autophagy · Ischemic Preconditioning · Cardioprotection - Myocardial Ischemia/Reperfusion

\section{Introduction}

There is increasing evidence that the induction of autophagy is associated with attenuation of ischemia/reperfusion (I/R) injury [1]. A number of known cardioprotective interventions have subsequently been shown to trigger autophagy, including rapamycin [2], caloric restriction [3], exercise [4], nitric oxide [5], and lipopolysaccharide [6]. A number of different pharmacologic agents have been shown to mimic ischemic preconditioning to achieve cardioprotection, including adenosine and various adenosine receptor agonists, purinergic receptor agonists such as UTP, the mitochondrial ATPsensitive potassium channel ( mitoK $_{\text {ATP }}$ ), and various cytochrome P450 inhibitors (reviewed in [7]). We previously demonstrated a requirement for autophagy in cardioprotection mediated by the adenosine A1 agonist 2-chlorocyclopentyladenosine (CCPA) and the cytochrome P450 inhibitor sulfaphenazole $[8,9]$. Despite a reported association between IPC and autophagy [10-12], whether autophagy is an essential mediator of protection conferred by IPC remains to be established. 
Macroautophagy (hereafter referred to as autophagy) is a cellular housekeeping process essential for removal of protein aggregates and damaged or unwanted organelles [13]. The process involves two parallel ubiquitin ligase-like systems: Atg12 must be conjugated onto Atg5; this step is essential for the subsequent conjugation of LC3 onto phosphatidylethanolamine in the growing autophagosomal membrane (reviewed in [7]). The coordinate action of these systems results in formation of autophagosomes which are recruited to engulf targets through the action of adapter proteins such as p62. The mature autophagosome transports its cargo to the lysosome for degradation and export of the breakdown products which include amino acids, carbohydrates, nucleic acids, and fatty acids. A variety of signals can induce autophagy, including protein kinase $\mathrm{C}$, reactive oxygen species (ROS), nitric oxide (NO), and AMPK. AMPK can inhibit mTOR, which is a powerful negative regulator of autophagy. Many of the signal transduction pathways that trigger autophagy appear to overlap with those known to signal preconditioning. For these reasons, we decided to investigate whether ischemic preconditioning would induce autophagy and whether autophagy is required for cardioprotection mediated by preconditioning.

\section{Materials and Methods}

In Vivo Studies of Autophagy and Preconditioning We used transgenic mice expressing the fusion protein mCherry-LC3 under the alpha myosin heavy chain promoter to study autophagy in vivo. We have previously described these mice $[8,9,14,15]$. Anesthetized mCherry-LC3 transgenic mice were subjected to regional ischemic preconditioning (three cycles of $5 \mathrm{~min}$ ischemia alternating with $5 \mathrm{~min}$ reperfusion); $1 \mathrm{~h}$ later, hearts were removed and cryosectioned. To quantitate autophagosomes, cryosections were washed with PBS for 5 min then briefly fixed with $4 \%$ paraformaldehyde. Using the $60 \times$ lens, mCherryLC 3 red dots were counted, and density per unit area in ten fields were determined. Three hearts were analyzed per condition.

Langendorff Perfusion The isolated perflused rat heart model was utilized as previously described [16, 17]. In brief, after anesthesia and heparinization (pentobarbital sodium $60 \mathrm{mg} / \mathrm{kg}$ i.p. and heparin $500 \mathrm{U}$ i.p.), rat hearts were excised into ice cold Krebs-Henseleit solution and perfused with oxygenated buffer within $30 \mathrm{~s}$. Hearts were perfused with buffer at $37^{\circ} \mathrm{C}$ at constant pressure $(60 \mathrm{mmHg})$ for $5 \mathrm{~min}$ for stabilization. Where indicated, Tat-ATG5 $^{\mathrm{K} 130 \mathrm{R}}(\sim 200 \mathrm{nM})$ or Tat-beta-galactosidase (Tat$\beta$-gal, $\sim 200 \mathrm{nM}$ ) was administered for $15 \mathrm{~min}$, followed by a buffer change before ischemic preconditioning $(3 \times 5 \mathrm{~min}$ global no-flow ischemia). Tat-ATG5 ${ }^{\mathrm{K} 130 \mathrm{R}}$ was prepared by cloning ATG5 ${ }^{\mathrm{K} 130 \mathrm{R}}$ into the pHA-Tat construct previously described [18]. Recombinant protein was purified as previously described [18-20]. For determination of autophagy, hearts were processed immediately after the IPC treatment. In other studies, following protein infusion and/ or IPC, global no-flow ischemia was maintained for $120 \mathrm{~min}$. CK release was measured during the first 15 min of reperfusion using the CK EC 2.7.3.2 UV test kit (Stanbio Laboratory, Boerne, TX, USA). After 2$\mathrm{h}$ reperfusion, infarct size was assessed by triphenyl tetrazolium chloride staining [17]. Other biochemical analyses of ischemic and reperfused heart tissue were performed on hearts flash-frozen in liquid nitrogen at the times indicated. All procedures were approved by the Animal Care and Use Committee at San Diego State University and conform to the Guide for the Care and Use of Laboratory Animals (National Institutes of Health publication no. 85-23, revised 1996).

Isolation of Protein Fractions Frozen heart samples were thawed on ice in homogenization buffer containing (in mmol/L) Tris- $\mathrm{HCl} 50, \mathrm{NaCl} 100$, EDTA 1, EGTA 1, 1\% Triton X-100, and protease inhibitor cocktail (Roche)). The tissue was minced and Polytron homogenized (Kinematica, Basel, Switzerland) on ice for $15 \mathrm{~s}$ for three passes. The homogenates were centrifuged at $600 \mathrm{~g}$ for $5 \mathrm{~min}$ at $4^{\circ} \mathrm{C}$, and the supernatant was centrifuged at $10,000 \mathrm{~g}$ for $10 \mathrm{~min}$ at $4^{\circ} \mathrm{C}$. The supernatant, designated as crude cytosol, was divided with aliquots stored at $-80^{\circ} \mathrm{C}$.

Western Blot Analysis Proteins prepared from rat hearts were quantified by Bio-Rad protein assay. For immunodetection, $50 \mu \mathrm{g}$ of crude cytosol prepared as above were resolved on SDS-PAGE 10\% denaturing gels and transferred to PVDF nylon membranes. The membranes were blocked with $5 \%$ nonfat dry milk in TNT buffer (in $\mathrm{mM}$ : $\mathrm{NaCl} 100$, Tris- $\mathrm{HCl} 10$ (pH 7.4), and 0.1\% Tween-20) for $1 \mathrm{~h}$. The blots were then incubated with 200 -fold diluted primary antibodies against LC3B-II (Cell Signaling Technology, Beverly, MA, USA) and p62 (BD Biosciences, San Jose, CA, USA) at $4^{\circ} \mathrm{C}$ overnight and then washed with TNT buffer at room temperature and incubated with appropriate peroxidase-conjugated secondary antibody (1:2,000 dilution). Immunoreactive bands were visualized by chemiluminescence (ECL kit, Amersham, Indianapolis, IN, USA) on X-ray film. Each immunoblotting experiment was repeated three to five times, and the results were averaged. To quantify the protein, intensity of bands was assessed with Scion Image Software.

Measurement of Autophagy by Cadaverine Uptake Langendorff-perfused rat hearts were minced in homogeni- 
zation buffer (250 $\mathrm{mM}$ Sucrose, $1 \mathrm{mM} \mathrm{Na} \mathrm{Na}_{2}$ EDTA, $10 \mathrm{mM}$ HEPES, pH 7.0, plus fresh protease inhibitors) and homogenized by Polytron for $5 \mathrm{~s}$ at half speed. Nuclei and heavy membranes were removed by centrifugation at $1,000 \times \mathrm{g}$ for $5 \mathrm{~min}$ at $4^{\circ} \mathrm{C}$. The post-nuclear supernatant was moved to new $1.5 \mathrm{~mL}$ centrifuge tubes and incubated with Alexa Fluor $488^{\mathrm{TM}}$ Cadaverine (Molecular Probes, Carlsbad, CA, USA) at $25 \mu \mathrm{M}$ final concentration for $10 \mathrm{~min}$. The samples were spun at $20,000 \times \mathrm{g}$ for $20 \mathrm{~min}$ at $4^{\circ} \mathrm{C}$ and the pellet washed twice with resuspension buffer $\left(140 \mathrm{mM} \mathrm{KCl}, 10 \mathrm{mM} \mathrm{MgCl} 2,5 \mathrm{mM} \mathrm{KH} \mathrm{PO}_{4}, 1 \mathrm{mM}\right.$ EGTA, $10 \mathrm{mM}$ MOPS, pH 7.4 plus fresh protease inhibitors). The pellet was resuspended in $350 \mu \mathrm{L}$ resuspension buffer and the fluorescence intensity read on a 96well plate reader at excitation/emission $495 / 519 \mathrm{~nm}$ in triplicate. The relative fluorescence units were standardized to the protein concentration of each sample which was determined by Bradford assay (Pierce, Rockford, IL, USA).

HL-1 Cell Culture Cells of the murine atrial-derived cardiac cell line HL-1 [21] were plated in gelatin/fibronectin-coated culture vessels and maintained in Claycomb medium [21] (JRH Biosciences, Lenexa, KS, USA) supplemented with $10 \%$ fetal bovine serum, $0.1 \mathrm{~mm}$ norepinephrine, $2 \mathrm{~mm}$ L-glutamine, $100 \mathrm{U} \mathrm{mL}^{-1}$ penicillin, $100 \mathrm{UmL}^{-1}$ streptomycin, and $0.25 \mu \mathrm{g} \mathrm{mL}^{-1}$ amphotericin B. HL-1 cells were transfected with the indicated vectors using the transfection reagent Effectene (Qiagen, Valencia, CA, USA), according to the manufacturer's instructions, achieving at least $40 \%$ transfection efficiency, as scored by the percentage of cells expressing GFP-LC3 (either diffuse or punctate distribution). The dominant negative pmCherryATG ${ }^{\mathrm{K} 130 \mathrm{R}}$ was previously described [22] and has been deposited with Addgene (www.addgene.org). For adult cardiomyocytes, GFP-LC3 infected cells were incubated with recombinant Tat-ATG5 ${ }^{\mathrm{K} 130 \mathrm{R}}$ for 30 min before adding $50 \mu \mathrm{M}$ UTP, $100 \mu \mathrm{M}$ DZX, or $1 \mu \mathrm{M}$ ranolazine. For simulated ischemia/reperfusion (sI/R) studies, cells were plated in 14-mm diameter glass bottom microwell dishes (MatTek, Ashland, MA, USA), and ischemia was introduced by a buffer exchange to ischemia-mimetic solution (in mM: 20 deoxyglucose, $125 \mathrm{NaCl}, 8 \mathrm{KCl}, 1.2 \mathrm{KH}_{2} \mathrm{PO}_{4}$, $1.25 \mathrm{MgSO}_{4}, 1.2 \mathrm{CaCl}_{2}, 6.25 \mathrm{NaHCO}_{3}, 5$ sodium lactate, 20 HEPES, pH 6.6) and placing the dishes in hypoxic pouches $\left(\mathrm{GasPak}^{\mathrm{TM}} \mathrm{EZ}\right.$, BD Biosciences, San Jose, CA, USA) equilibrated with $95 \% \mathrm{~N}_{2}, 5 \% \mathrm{CO}_{2}$. After $2 \mathrm{~h}$ of simulated ischemia, reperfusion was initiated by a buffer exchange to normoxic MKH buffer and incubation at $95 \%$ room air, $5 \% \mathrm{CO}_{2}$. Controls incubated in normoxic $\mathrm{MKH}$ buffer were run in parallel for each condition for periods of time that corresponded with those of the experimental groups.
To analyze autophagy in cell culture, cells were inspected at $60 \times$ magnification and classified as: (a) cells with predominantly diffuse GFP-LC3 fluorescence or as (b) cells with numerous GFP-LC3 puncta ( $>30$ dots/cell), representing autophagosomes. At least 200 cells were scored for each condition in three or more independent experiments. Protein content and LDH activity were determined according to El-Ani et al. [23]. Briefly, $25 \mu \mathrm{L}$ supernatants from $35 \mathrm{~mm}$ dishes were transferred into wells of a 96-well plate, and the LDH activities were determined with an LDH-L kit (Sigma, St. Louis, MO, USA), according to the manufacturer. The product of the enzyme was measured spectrophotometrically at $30^{\circ} \mathrm{C}$ at a wavelength of $340 \mathrm{~nm}$ as described previously [24]. The results were expressed relative to the control (X-fold) in the same experiment. Each experimental condition was done in triplicate, and the experiments were repeated at least three times.

Statistical Analysis Statistical analysis was performed between groups by ANOVA by using INSTAT 4.10 software (GraphPad, La Jolla, CA, USA). A $P$ value $<0.05$ was considered significant.

\section{Results}

Ischemic Preconditioning Induces Autophagy

To assess autophagy, mCherry-LC3 transgenic mice were anesthetized and subjected to $3 \times 5 \mathrm{~min}$ LAD ligation to induce IPC. One hour later, hearts were removed and autophagy was assessed by fluorescence microscopy. IPC increased the number of mCherry-LC3labeled autophagosomes in the area at risk (Fig. 1a and b). We also measured autophagy by the cadaverine dye binding assay. As shown in Fig. 1c, IPC resulted in increased levels of dye binding, consistent with upregulation of autophagy.

We then assessed autophagy in the Langendorff model. Isolated perfused rat hearts were subjected to $3 \times 5 \mathrm{~min}$ global no-flow ischemia alternating with reperfusion. After the third cycle, autophagy was measured by cadaverine dye binding and Western blot for p62, a key autophagy protein which is known to be upregulated in response to oxidative stress [25]. Although p62 accumulates in tissues in association with ubiquitinated protein aggregates when autophagy is chronically suppressed [26], protein levels of p62 can rise acutely in response to oxidative stress or other stimuli [25]. We have observed an acute (within $10 \mathrm{~min}$ ) upregulation of Beclin1 and LC3, as well as p62, in hearts subjected to a pharmacologic preconditioning agent (unpublished data). Autophagy was increased immediately 
Fig. 1 Autophagy is induced by IPC in vivo. a mCherry-LC3 mice were subjected to three cycles of 5 min LAD occlusion alternating with 5 min reperfusion. One hour later, hearts were harvested and cryosections were prepared. b Quantification of autophagosomes (red puncta) from cryosections of shamoperated hearts, preconditioned hearts in the risk regions $(I P C)$, and in the nonrisk (non-IPC) regions. Ten representative fields were scored for three hearts per condition $(P<0.05$, $N=3)$. c. Quantification of autophagy by cadaverine dyebinding assay $(P<0.05, N=3)$

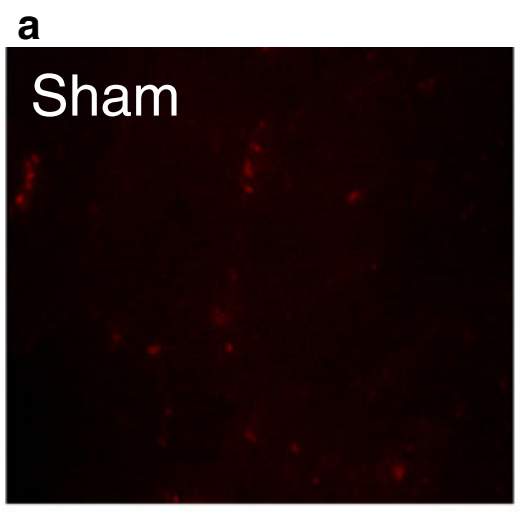

\section{b}
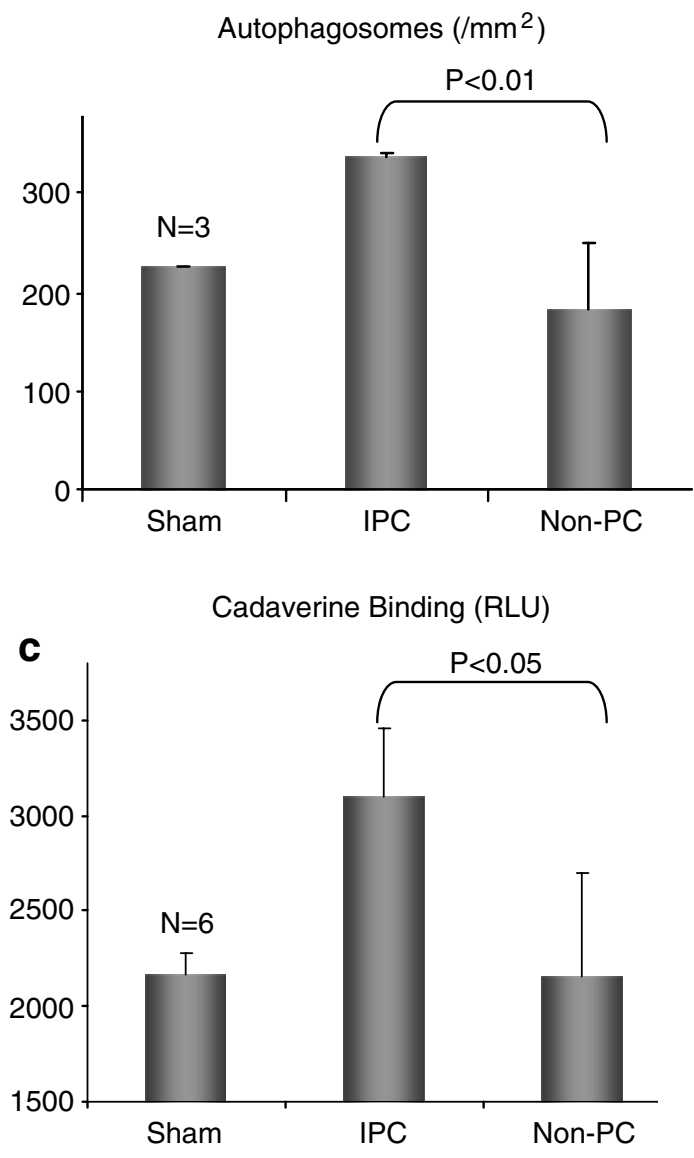

after IPC, as indicated by increased cadaverine dye binding (Fig. 2a) and increased p62 protein (Fig. 2b).

\section{Autophagy is Required for Cardioprotection by IPC}

In order to establish the role of autophagy in IPC-mediated cardioprotection, we used the specific inhibitor of autophagy, Tat-ATG $5^{\mathrm{K} 130 \mathrm{R}}$ in Langendorff-perfused rat hearts. Tat- $\beta$-gal was used as a protein control. Hearts were treated for $15 \mathrm{~min}$ and then subjected to IPC $(3 \times 5 \mathrm{~min}$ global noflow ischemia alternating with reperfusion) followed by 30 min global no-flow ischemia and $2 \mathrm{~h}$ reperfusion (Fig. 3a). Control hearts subjected to $I / R$ had infarcts averaging $42 \%$ of the area at risk (Fig. 3b). Hearts subjected to IPC in the presence of the control protein (Tat- $\beta$-gal) were protected against $\mathrm{I} / \mathrm{R}$ injury, with an average infarct size of $20 \%$ (Fig. 3b). In contrast, hearts pretreated with the autophagy inhibitor Tat-ATG $5^{\mathrm{K} 130 \mathrm{R}}$ had larger infarcts, indicating that IPC was significantly less effective when autophagy was inhibited. In the absence of IPC, Tat-ATG $5^{\mathrm{K} 130 \mathrm{R}}$ had no effect on infarct size.

Autophagy is Involved in Cytoprotection Mediated by Diazoxide, Utp, and Ranolazine

We selected three structurally diverse cardioprotective agents to ascertain their ability to induce autophagy in cardiomyocytes. We previously reported that HL-1 cells behave similarly to adult rat cardiomyocytes in response to simulated $\mathrm{I} / \mathrm{R}$ and the cardioprotective agents CCPA and sulfaphenazole [8,9]. Diazoxide, UTP, and ranolazine all increased autophagy rapidly (Figs. 4a and 5a). Transient transfection with ATG $5^{\mathrm{K} 130 \mathrm{R}}$ suppressed autophagy induced by these agents (Fig. 4a). To determine the requirement for autophagy in cytoprotection by these agents, we transfected HL-1 cells with $\mathrm{ATG} 5^{\mathrm{K} 130 \mathrm{R}}$ and $24 \mathrm{~h}$ later subjected them to sI/R in the 
a

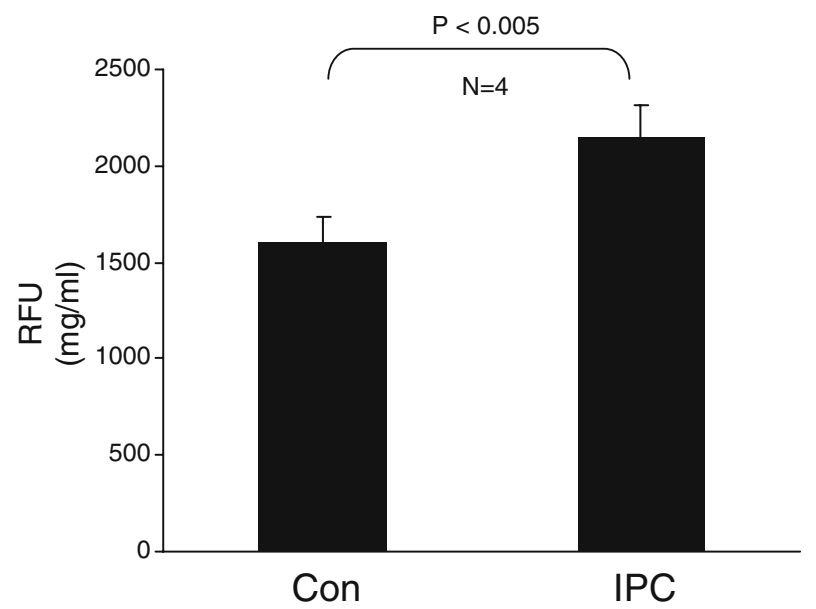

b
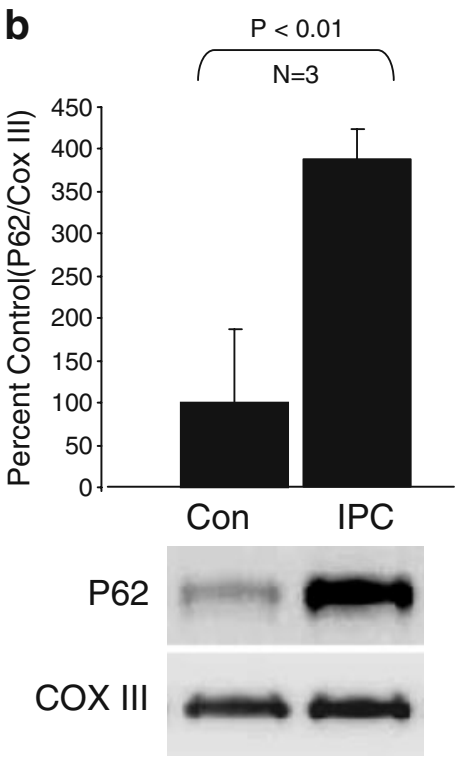

Fig. 2 Autophagy is induced by IPC in the rat Langendorff model. a Quantification of autophagy by cadaverine dye-binding assay. Hearts underwent three cycles of 5 min global no-flow ischemia alternating with 5 min reperfusion (IPC) or continuous perfusion for the same duration of time (Con). Samples were processed immediately after the

presence or absence of the cardioprotective drugs. As shown in Fig. 4b, inhibition of autophagy by ATG $5^{\mathrm{K} 130 \mathrm{R}}$ abolished cytoprotection by ranolazine, UTP, and diazoxide, agents which elicit cytoprotection via diverse signaling pathways. third cycle of reperfusion $(P<0.005, N=4)$. b Upregulation of autophagy marker p62 by IPC. Densitometric quantitation of p62 normalized to the mitochondrial protein cytochrome oxidase subunit III $(C O X$ III $)$ is shown as relative to control hearts $(P<0.01, N=3)$. A representative immunoblot of $\mathrm{p} 62$ and COX III is shown

Specifically, treatment with ATG $5^{\mathrm{K} 130 \mathrm{R}}$ prior to exposing the cells to the cardioprotective drugs resulted in LDH release levels comparable to that associated with ischemia alone. Similarly, studies in adult cardiomyocytes infected with

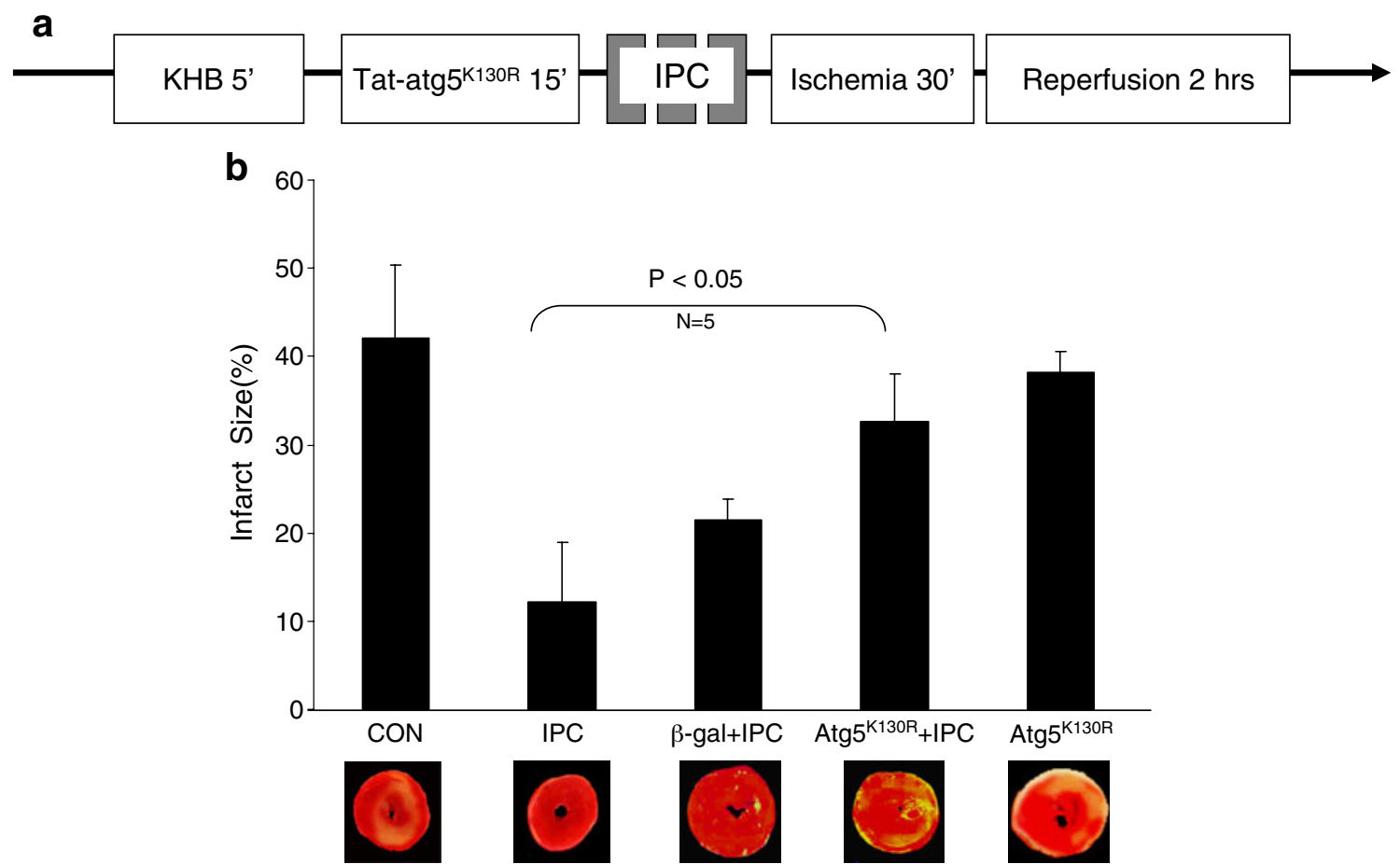

Fig. 3 Inhibition of autophagy reduces cardioprotection. a Protocol for Langendorff perfusion. After stabilization, protein (TAT- $\mu$-gal or Tat-ATG5 ${ }^{\mathrm{K} 130 \mathrm{R}}$ ) was infused for $15 \mathrm{~min}$. Hearts then underwent three cycles of $5 \mathrm{~min}$ global no-flow ischemia alternating with $5 \mathrm{~min}$ reperfusion $(I P C)$ followed by $30 \mathrm{~min}$ of ischemia and $2 \mathrm{~h}$ reperfusion. b Infarct size measured from TTC-stained heart sections is shown $(P<$ $0.05, N=5$ ) 
a

$\square$ Con
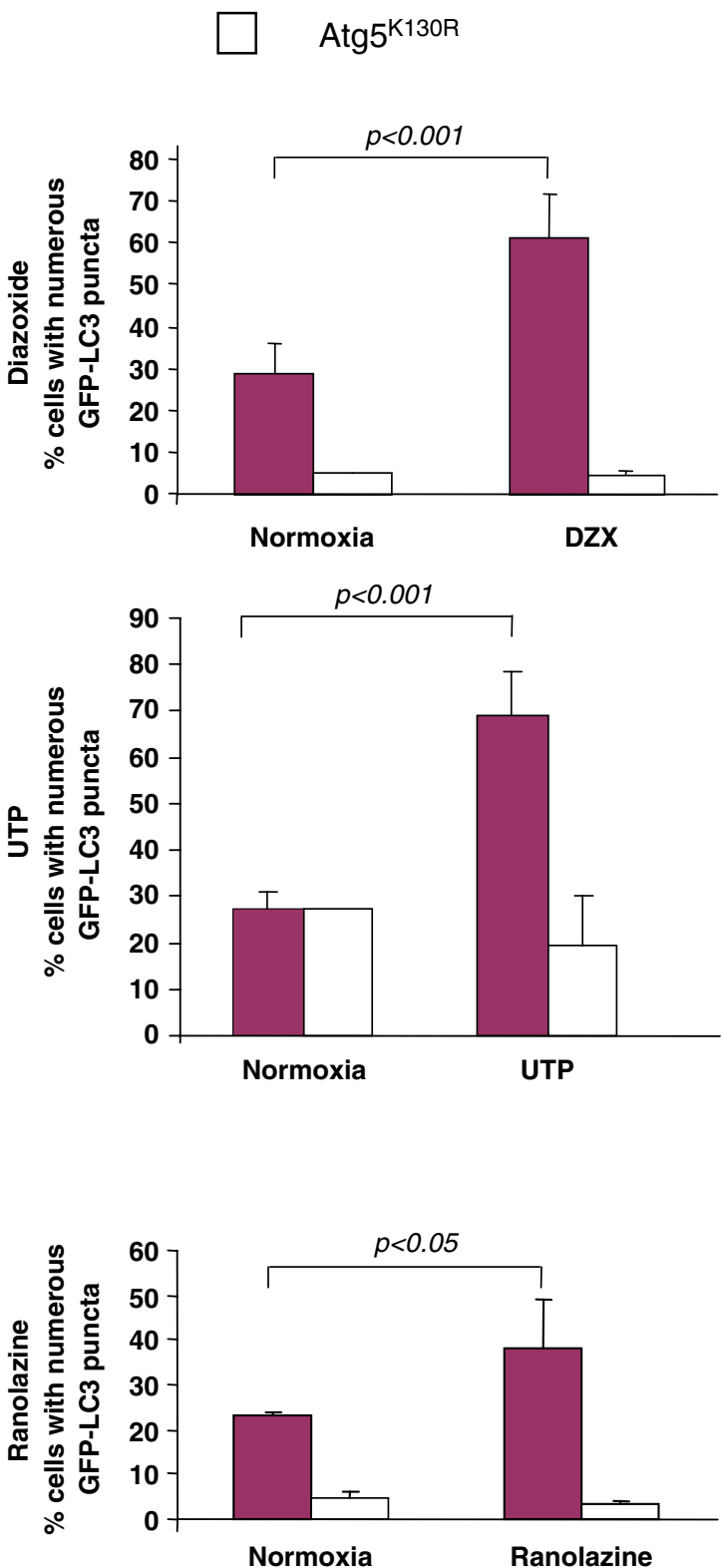

Fig. 4 Cardioprotective agents involve autophagy in HL-1 cells. a Autophagy was scored in GFP-LC3-transfected HL-1 cells exposed to the indicated agents for $10 \mathrm{~min}$. Where indicated, cells were cotransfected with the autophagy inhibitor ATG $5^{\mathrm{K} 130 \mathrm{R}}$. b HL-1 cells

adenovirus for GFP-LC3 revealed that CCPA, UTP, and diazoxide induced autophagy which was suppressed by Tat$\mathrm{ATG} 5^{\mathrm{K} 130 \mathrm{R}}$ (Fig. 5b).

\section{Discussion}

We studied the role of autophagy in ischemic preconditioning. Ischemic preconditioning involves intracellular signaling through G-protein coupled receptors and a diverse array of
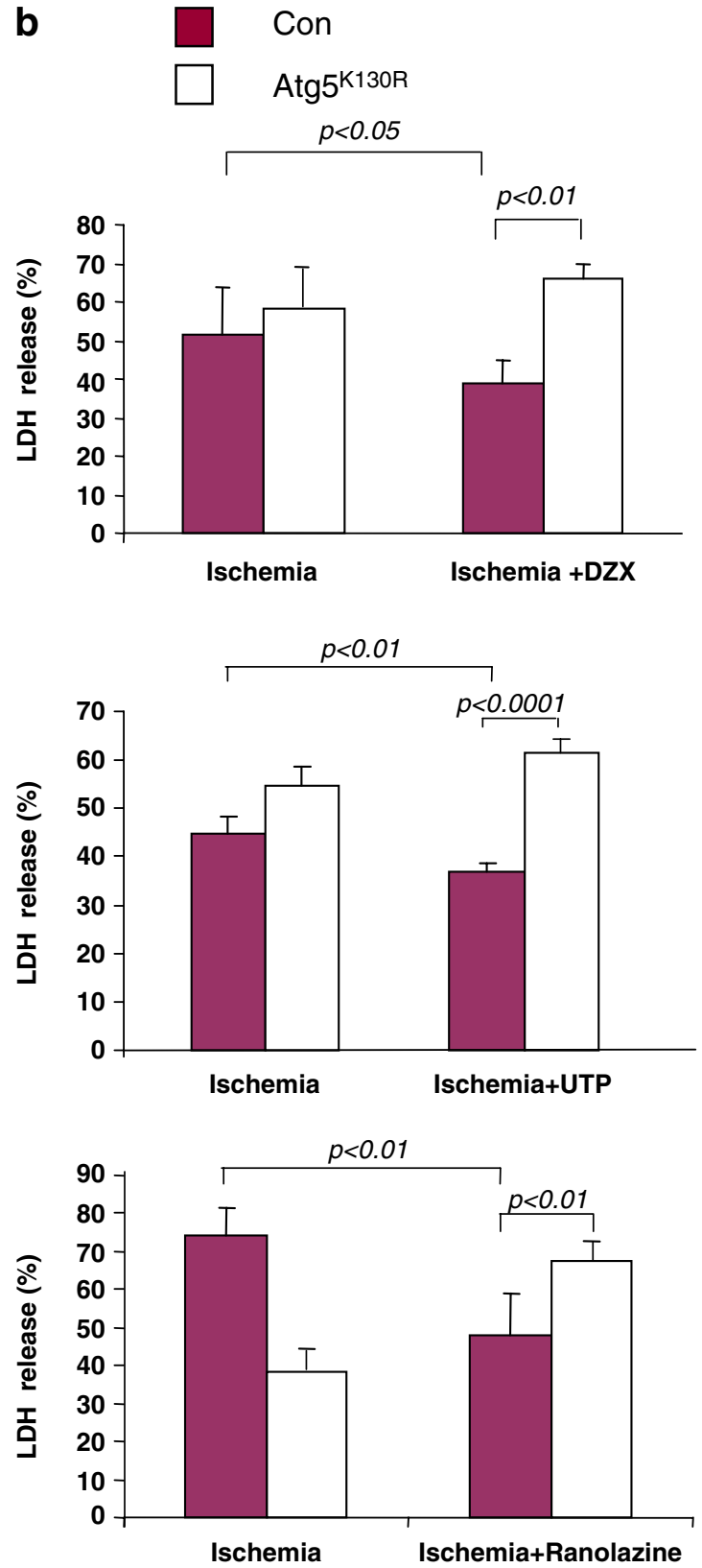

were transfected with GFP-LC3 and with or without ATG5 $5^{\mathrm{K} 130 \mathrm{R}}$ then subjected to simulated ischemia in the presence or absence of the indicated drug. At the end of 2-h simulated ischemia, cell culture supernatant was harvested for LDH determination

protein kinases including protein kinase C and Akt [27]. The putative mitochondrial ATP-sensitive potassium channel (mitoK ${ }_{\mathrm{ATP}}$ ), production of nitric oxide, and ROS are also consistent features of IPC $[5,28]$. To extend the potential significance of autophagy in cardioprotection beyond ischemic preconditioning, we also examined three structurally unrelated cardioprotective agents, which are as follows: UTP [29], diazoxide [30], and ranolazine [31]. UTP binds to a purinergic receptor that shares downstream signaling with adenosine [32]. Diazoxide has been suggested to directly 

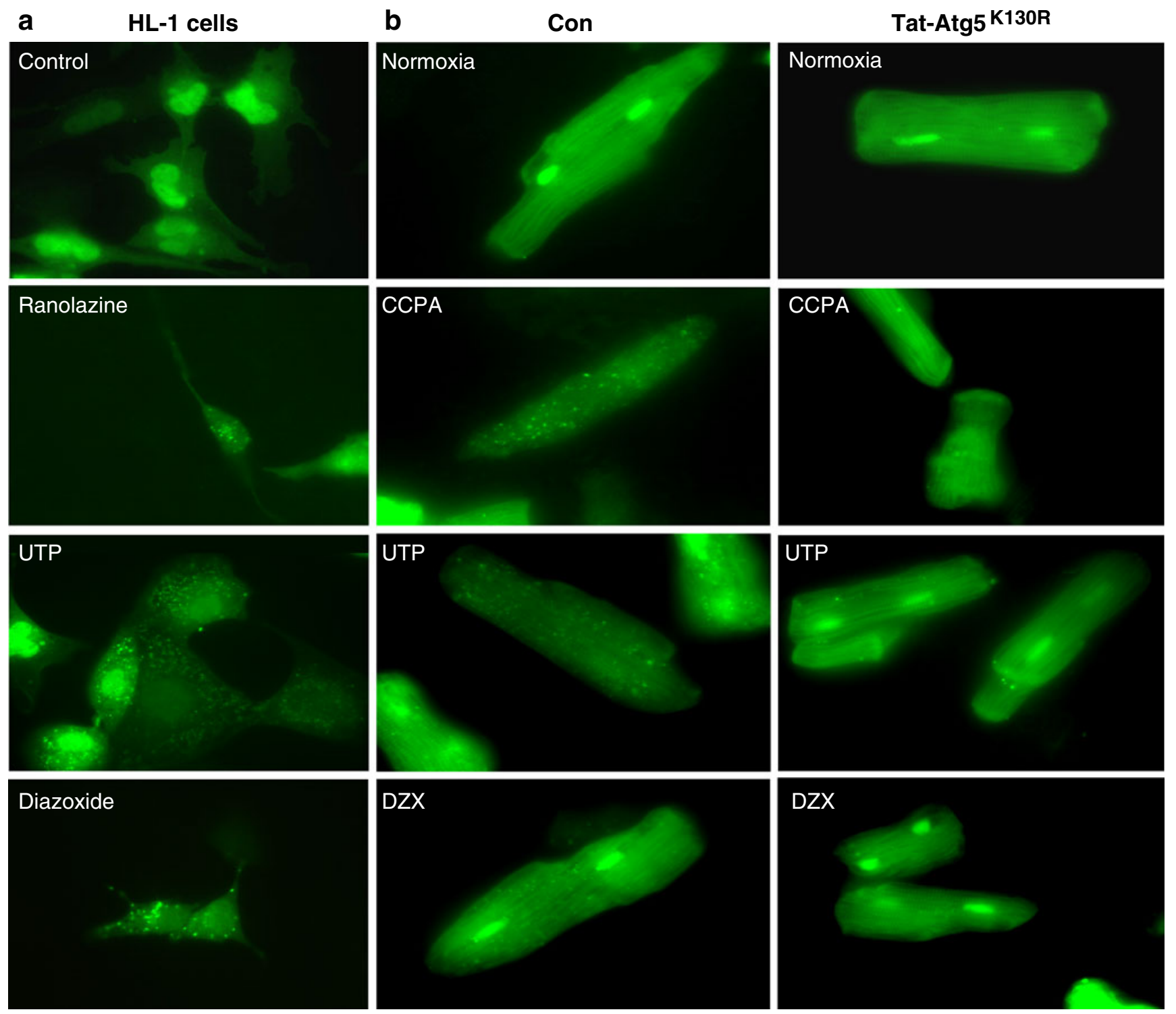

Fig. 5 Modulation of autophagy by pharmacologic agents and TatATG5 ${ }^{\text {K130R }}$. a HL-1 cells were transfected with GFP-LC3 and treated with the indicated drugs for $10 \mathrm{~min}$. Representative images of treated cells are shown. b Adult rat cardiomyocytes were infected with

open the mitoK $_{\mathrm{ATP}}$, accompanied by a modest increase in ROS production and activation of protein kinase $\mathrm{C}$ [33]. Ranolazine is a sodium channel inhibitor that also interferes with fatty acid oxidation and may have unknown effects on other cardioprotective signaling pathways [34, 35]. In the present study, we demonstrate that ischemic preconditioning is associated with increased autophagosome formation in vivo and ex vivo. We also demonstrate the upregulation of autophagy by UTP, diazoxide, and ranolazine, although the signal transduction pathway leading to autophagy remains to be elucidated. Our study demonstrates that inhibition of autophagy attenuates their protective effects, and supports the concept that autophagy is an important element of cardioprotection. adenovirus for GFP-LC3 and treated with or without Tat-ATG5 $5^{\mathrm{K} 130 \mathrm{R}}$ before adding the indicated drugs for $10 \mathrm{~min}$. Representative images of cells are shown

Autophagy has long been recognized to occur in cardiac tissue. Decker and Wildenthal observed that induction of autophagy correlated with functional recovery of the rabbit heart after I/R and noted that prolonged ischemia appeared to impair the autophagosome-lysosomal pathway, which correlated with irreversible damage and contractile dysfunction [36]. Vatner's group reported increased expression of several autophagy-related genes in the chronically ischemic myocardium [37] and suggested it might protect against apoptosis. Subsequently, several groups have shown that autophagy is increased in the setting of ischemic or pharmacologic preconditioning [8-12, 38] and have suggested that it serves a protective function. Interestingly, a number of agents that have been shown to be cardioprotective may also involve the 
induction of autophagy, including rapamycin [2, 22], hydrophobic statins [39, 40], lipopolysaccharide [6, 14, 41], and chloramphenicol [42]. The requirement for autophagy in cardioprotection has been established for the adenosine A1 agonist CCPA [8], the cytochrome P450 inhibitor sulfaphenazole [9], and in this study, IPC.

Direct demonstration of a requirement for autophagy in preconditioning has been limited by the lack of specific tools to inhibit autophagy. In order to overcome this and definitively establish the requirement for autophagy, we used a cell-permeable agent to specifically inhibit autophagy, Tat-ATG5 ${ }^{\mathrm{K} 130 \mathrm{R}}$. We previously showed that Tat$\mathrm{ATG} 5^{\mathrm{K} 130 \mathrm{R}}$ effectively inhibits autophagy in the isolated perfused heart model and partially abrogated cardioprotection by sulfaphenazole [9]. In this study, we again observed a partial loss of protection by preconditioning in the Tat$\mathrm{ATG} 5^{\mathrm{K} 130 \mathrm{R}}$-infused hearts. This raises the possibility of an unrelated mechanism of protection.

The stressed heart may benefit from amino acids, carbohydrates, and fatty acids generated by autophagic degradation of cellular proteins, lipids, and glycogen granules. Several studies have shown that blocking lysosomal fusion in fasted animals result in plummeting ATP levels in the heart, suggesting that production of metabolic substrates is important, at least in the setting of fasting [43]. Autophagy might also improve ion homeostasis by exporting protons via vacuolar proton ATPase pumping into lysosomes. This would limit the need for $\mathrm{Na}^{+} / \mathrm{H}^{+}$exchange and would thereby limit secondary $\mathrm{Ca}^{++}$overload (via $\mathrm{Na}^{+} / \mathrm{Ca}^{++}$ exchange). Consistent with this notion, we previously showed that inhibition of the VPATPase disrupted cytoprotection and ion homeostasis associated with ischemic preconditioning [44, 45]. Limiting calcium overload might avoid triggering the mitochondrial permeability transition, a critical phenomenon leading to ischemic cell death.

The heart is critically dependent on autophagy for normal homeostasis as well as the response to stress. Our results reveal autophagy to be an important element of the endogenous defense mechanisms activated by ischemic preconditioning. Identification of agents that can rapidly induce autophagy could lead to novel cardioprotective drugs. IPC and pharmacologic agents have shown promise in animal studies, but many have failed in the clinical setting [46]. In part, this may be due to the fact that most preclinical studies use young, healthy animals, whereas clinical studies involve patients with underlying diseases including metabolic syndrome, diabetes, hypertension, and advanced age. Many of these conditions have been shown to interfere with autophagy [47], which could explain the failure of preconditioning agents in patients. In order to be efficacious, a candidate cardioprotective drug would also need to induce autophagy in the target population, with the appropriate timing and magnitude of response. The key finding of our study is that autophagy appears to be a common feature of cardioprotection conferred by ischemic preconditioning and a variety of pharmacologic conditioning agents. Further work is needed to understand the mechanistic basis of cardioprotection by autophagy.

Acknowledgements This work was supported by NIH R01 HL071091 and R01 AG033283 (to RAG) and R01 HL034579 (to RMM).

Open Access This article is distributed under the terms of the Creative Commons Attribution Noncommercial License which permits any noncommercial use, distribution, and reproduction in any medium, provided the original author(s) and source are credited.

\section{References}

1. Gottlieb, R. A., Finley, K. D., \& Mentzer, R. M., Jr. (2009). Cardioprotection requires taking out the trash. Basic Research in Cardiology, 104, 169-180.

2. Khan, S., Salloum, F., Das, A., Xi, L., Vetrovec, G. W., \& Kukreja, R. C. (2006). Rapamycin confers preconditioning-like protection against ischemia-reperfusion injury in isolated mouse heart and cardiomyocytes. Journal of Molecular and Cellular Cardiology, 41, 256-264.

3. Marzetti E., Wohlgemuth S.E., Anton S.D., Bernabei R., Carter C. S., Leeuwenburgh C. (2009). Cellular mechanisms of cardioprotection by calorie restriction: state of the science and future perspectives. Clin Geriatr Med, 25, 715-32, ix.

4. Kavazis, A. N., Alvarez, S., Talbert, E., Lee, Y., \& Powers, S. K. (2009). Exercise training induces a cardioprotective phenotype and alterations in cardiac subsarcolemmal and intermyofibrillar mitochondrial proteins. American Journal of Physiology. Heart and Circulatory Physiology, 297, H144-H152.

5. Jones, S. P., \& Bolli, R. (2006). The ubiquitous role of nitric oxide in cardioprotection. Journal of Molecular and Cellular Cardiology, 40, 16-23.

6. Ha, T., Hua, F., Liu, X., Ma, J., McMullen, J. R., et al. (2008). Lipopolysaccharide-induced myocardial protection against ischaemia/reperfusion injury is mediated through a PI3K/Akt-dependent mechanism. Cardiovascular Research, 78, 546-553.

7. Gustafsson, A. B., \& Gottlieb, R. A. (2008). Eat your heart out: role of autophagy in myocardial ischemia/reperfusion. Autophagy, 4, 416-421.

8. Yitzhaki, S., Huang, C., Liu, W., Lee, Y., Gustafsson, A. B., et al. (2009). Autophagy is required for preconditioning by the adenosine A1 receptor-selective agonist CCPA. Basic Research in Cardiology, 104, 157-167.

9. Huang, C., Liu, W., Perry, C. N., Yitzhaki, S., Lee, Y., et al. (2010). Autophagy and protein kinase c are required for cardioprotection by sulfaphenazole. American Journal of Physiology. Heart and Circulatory Physiology, 298(2), H570-H579.

10. Yan, L., Sadoshima, J., Vatner, D. E., \& Vatner, S. F. (2009). Autophagy in ischemic preconditioning and hibernating myocardium. Autophagy, 5, 709-712.

11. Gurusamy, N., Lekli, I., Gorbunov, N., Gherghiceanu, M., Popescu, L. M., \& Das, D. K. (2009). Cardioprotection by adaptation to ischemia augments autophagy in association with BAG-1 protein. Journal of Cellular and Molecular Medicine, 13(2), 373-387.

12. Park, H. K., Chu, K., Jung, K. H., Lee, S. T., Bahn, J. J., et al. (2009). Autophagy is involved in the ischemic preconditioning. Neuroscience Letters, 451, 16-19. 
13. Dengjel, J., Kristensen, A. R., \& Andersen, J. S. (2008). Ordered bulk degradation via autophagy. Autophagy, 4, 1057-1059.

14. Yuan, H., Perry, C. N., Huang, C., Iwai-Kanai, E., Carreira, R. S., et al. (2009). LPS-induced autophagy is mediated by oxidative signaling in cardiomyocytes and is associated with cytoprotection. American Journal of Physiology. Heart and Circulatory Physiology, 296, H470-H479.

15. Iwai-Kanai, E., Yuan, H., Huang, C., Sayen, M. R., Perry-Garza, C. N., et al. (2008). A method to measure cardiac autophagic flux in vivo. Autophagy, 4, 322-329.

16. Granville, D. J., Tashakkor, B., Takeuchi, C., Gustafsson, A. B., Huang, C., et al. (2004). Reduction of ischemia and reperfusioninduced myocardial damage by cytochrome P450 inhibitors. Proceedings of the National Academy of Sciences of the United States of America, 101, 1321-1326.

17. Chen, M., Won, D. J., Krajewski, S., \& Gottlieb, R. A. (2002). Calpain and mitochondria in ischemia/reperfusion injury. The Journal of Biological Chemistry, 277, 29181-29186.

18. Becker-Hapak, M., McAllister, S. S., \& Dowdy, S. F. (2001). TAT-mediated protein transduction into mammalian cells. Methods, 24, 247-256.

19. Hamacher-Brady, A., Brady, N. R., Logue, S. E., Sayen, M. R., Jinno, M., et al. (2007). Response to myocardial ischemia/ reperfusion injury involves Bnip3 and autophagy. Cell Death and Differentiation, 14, 146-157.

20. Gustafsson, A. B., Sayen, M. R., Williams, S. D., Crow, M. T., \& Gottlieb, R. A. (2002). TAT protein transduction into isolated perfused hearts: TAT-apoptosis repressor with caspase recruitment domain is cardioprotective. Circulation, 106, 735-739.

21. Claycomb, W. C., Lanson, N. A., Jr., Stallworth, B. S., Egeland, D. B., Delcarpio, J. B., et al. (1998). HL-1 cells: a cardiac muscle cell line that contracts and retains phenotypic characteristics of the adult cardiomyocyte. Proceedings of the National Academy of Sciences of the United States of America, 95, 2979-2984.

22. Hamacher-Brady, A., Brady, N. R., \& Gottlieb, R. A. (2006). Enhancing macroautophagy protects against ischemia/reperfusion injury in cardiac myocytes. The Journal of Biological Chemistry, 281, 29776-29787.

23. El-Ani, D., Jacobson, K. A., \& Shainberg, A. (1994). Characterization of adenosine receptors in intact cultured heart cells. Biochemical Pharmacology, 48, 727-735.

24. Safran, N., Shneyvays, V., Balas, N., Jacobson, K. A., Nawrath, H., \& Shainberg, A. (2001). Cardioprotective effects of adenosine $\mathrm{A} 1$ and $\mathrm{A} 3$ receptor activation during hypoxia in isolated rat cardiac myocytes. Molecular and Cellular Biochemistry, 217, 143-152.

25. Ishii, T., Yanagawa, T., Kawane, T., Yuki, K., Seita, J., et al. (1996). Murine peritoneal macrophages induce a novel $60-\mathrm{kDa}$ protein with structural similarity to a tyrosine kinase p56(lck)associated protein in response to oxidative stress. Biochemical and Biophysical Research Communications, 226, 456-460.

26. Bjorkoy, G., Lamark, T., Pankiv, S., Overvatn, A., Brech, A., \& Johansen, T. (2009). Monitoring autophagic degradation of p62/ SQSTM1. Methods in Enzymology, 452, 181-197.

27. Downey, J. M., Krieg, T., \& Cohen, M. V. (2008). Mapping preconditioning's signaling pathways: an engineering approach. Annals of the New York Academy of Sciences, 1123, 187-196.

28. Pain, T., Yang, X. M., Critz, S. D., Yue, Y., Nakano, A., et al. (2000). Opening of mitochondrial K(ATP) channels triggers the preconditioned state by generating free radicals. Circulation Research, 87, 460-466.

29. Yitzhaki, S., Shainberg, A., Cheporko, Y., Vidne, B. A., Sagie, A., et al. (2006). Uridine-5'-triphosphate (UTP) reduces infarct size and improves rat heart function after myocardial infarct. Biochemical Pharmacology, 72, 949-955.
30. Garlid, K. D., Paucek, P., Yarov-Yarovoy, V., Murray, H. N., Darbenzio, R. B., et al. (1997). Cardioprotective effect of diazoxide and its interaction with mitochondrial ATP-sensitive $\mathrm{K}$ + channels. Possible mechanism of cardioprotection. Circulation Research, 81, 1072-1082.

31. McCormack, J. G., Barr, R. L., Wolff, A. A., \& Lopaschuk, G. D. (1996). Ranolazine stimulates glucose oxidation in normoxic, ischemic, and reperfused ischemic rat hearts. Circulation, 93, 135-142.

32. Yitzhaki, S., Shneyvays, V., Jacobson, K. A., \& Shainberg, A. (2005). Involvement of uracil nucleotides in protection of cardiomyocytes from hypoxic stress. Biochemical Pharmacology, 69, 1215-1223.

33. Wang, Y., Takashi, E., Xu, M., Ayub, A., \& Ashraf, M. (2001). Downregulation of protein kinase $\mathrm{C}$ inhibits activation of mitochondrial K(ATP) channels by diazoxide. Circulation, 104, 85-90.

34. Hale, S. L., Shryock, J. C., Belardinelli, L., Sweeney, M., \& Kloner, R. A. (2008). Late sodium current inhibition as a new cardioprotective approach. Journal of Molecular and Cellular Cardiology, 44, 954-967.

35. Folmes, C. D., Clanachan, A. S., \& Lopaschuk, G. D. (2005). Fatty acid oxidation inhibitors in the management of chronic complications of atherosclerosis. Current Atherosclerosis Reports, 7, 63-70.

36. Decker, R. S., \& Wildenthal, K. (1980). Lysosomal alterations in hypoxic and reoxygenated hearts. I. Ultrastructural and cytochemical changes. The American Journal of Pathology, 98, 425-444.

37. Yan, L., Vatner, D. E., Kim, S. J., Ge, H., Masurekar, M., et al. (2005). Autophagy in chronically ischemic myocardium. Proceedings of the National Academy of Sciences of the United States of America, 102, 13807-13812.

38. Gurusamy, N., Lekli, I., Gherghiceanu, M., Popescu, L. M., \& Das, D. K. (2009). BAG-1 induces autophagy for cardiac cell survival. Autophagy, 5, 120-121.

39. Araki, M., \& Motojima, K. (2008). Hydrophobic statins induce autophagy in cultured human rhabdomyosarcoma cells. Biochemical and Biophysical Research Communications, 367, 462-467.

40. Spin, J. M., \& Vagelos, R. H. (2003). Early use of statins in acute coronary syndromes. Current Atherosclerosis Reports, 5, 44-51.

41. Hickson-Bick, D. L., Jones, C., \& Buja, L. M. (2008). Stimulation of mitochondrial biogenesis and autophagy by lipopolysaccharide in the neonatal rat cardiomyocyte protects against programmed cell death. Journal of Molecular and Cellular Cardiology, 44, 411-418.

42. Sala-Mercado J.A., Wider J., Jahania S., Mentzer R.M., Jr, Gottlieb R.A., Przyklenk K. (2009). Abstract 3363: profound cardioprotection with chloramphenicol in the swine model of myocardial ischemiareperfusion injury. Circulation, 120, S795-b-.

43. Kanamori, H., Takemura, G., Maruyama, R., Goto, K., Tsujimoto, A., et al. (2009). Functional significance and morphological characterization of starvation-induced autophagy in the adult heart. The American Journal of Pathology, 174, 1705-1714.

44. Gottlieb, R. A., Gruol, D. L., Zhu, J. Y., \& Engler, R. L. (1996). Preconditioning in rabbit cardiomyocytes: Role of $\mathrm{pH}$, vacuolar proton ATPase, and apoptosis. The Journal of Clinical Investigation, 97, 2391-2398.

45. Karwatowska-Prokopczuk, E., Nordberg, J., Li, H. L., Engler, R. L., \& Gottlieb, R. A. (1998). Effect of the vacuolar proton ATPase on intracellular $\mathrm{pH}$, calcium, and on apoptosis in neonatal cardiomyocytes during metabolic inhibition and recovery. Circulation Research, 82, 1139-1144.

46. Ferdinandy, P., Schulz, R., \& Baxter, G. F. (2007). Interaction of cardiovascular risk factors with myocardial ischemia/reperfusion injury, preconditioning, and postconditioning. Pharmacological Reviews, 59, 418-458.

47. Bergamini, E., Cavallini, G., Donati, A., \& Gori, Z. (2004). The role of macroautophagy in the ageing process, anti-ageing intervention and age-associated diseases. The International Journal of Biochemistry \& Cell Biology, 36, 2392-2404. 